\title{
Dieta e comportamento de forrageamento de Suiriri affinis e $S$. islerorum (Aves, Tyrannidae) em um cerrado do Brasil central
}

\author{
Leonardo E. Lopes
}

Pós-graduação em Ecologia, Conservação e Manejo da Vida Silvestre, Lab. de Ornitologia, Depto. de Zoologia, ICB, Universidade Federal de Minas Gerais, Av. Antônio Carlos, 6627, Pampulha, 31270-910 Belo Horizonte, MG, Brasil. (leo.cerrado@gmail.com)

\begin{abstract}
Diet and foraging behavior of Suiriri affinis and S. islerorum (Aves, Tyrannidae) in a central Brazilian cerrado. The foraging behavior of Suiriri affinis (Campo Suiriri) and S. islerorum (Chapada Flycatcher), two sintopic Tyrant Flycatchers in the Central Brazilian Cerrado (tropical savanna) was studied. During 2003, 188 feeding bouts of S. affinis and 150 of S. islerorum were registered. Differences between the two species in the feeding height, foraging substrate, phytophysiognomy used, and foliage density at the point of foraging observation were found. The attack methods, attack direction, distance of flight, and plant species used did not differ between both species. The analysis of 26 stomach contents of members of this genus revealed only arthropods. The low percentage of feeding bouts directed to fruits (only $7 \%$ and $9 \%$, respectively) confirmed the insectivorous character of this genus.
\end{abstract}

KEYWORDS. Diet, foraging behavior, Suiriri affinis, Suiriri islerorum, Cerrado.

RESUMO. Foi estudado o comportamento de forrageamento de Suiriri affinis (suiriri-do-cerrado) e S. islerorum (suiriri-da-chapada), duas espécies de Tyrannidae que ocorrem em sintopia nos cerrados do Brasil central. Durante o ano de 2003 foram registradas 188 observações de forrageamento para $S$. affinis e 150 para $S$. islerorum. Foram encontradas diferenças significativas entre as duas espécies em relação à altura e substrato de forrageamento, fitofisionomia utilizada e densidade da folhagem no local do ataque. $\mathrm{O}$ comportamento e a direção do ataque, a espécie de planta e a distância de vôo não diferiram entre as espécies. A análise de 26 conteúdos estomacais de membros do gênero revelou o consumo exclusivo de artrópodes. A baixa porcentagem de observações de forrageamento direcionadas a frutos (apenas $7 \%$ e $9 \%$, respectivamente) confirmou o caráter insetívoro do gênero.

PALAVRAS-CHAVE. Dieta, comportamento de forrageamento, Suiriri affinis, Suiriri islerorum, Cerrado.

Na região central do Brasil, no domínio do Cerrado, ocorrem duas espécies do gênero Suiriri (Aves: Tyrannidae). Suiriri islerorum Zimmer, Whittaker \& Oren 2001 foi descrita recentemente (ZIMMER et al., 2001), tendo sido até então confundida com a outra espécie, $S$. affinis (Burmeister, 1856). Suiriri islerorum encontra-se amplamente distribuído pelo Cerrado, sendo considerado endêmico deste bioma (SILVA \& Bates, 2002; L. E. Lopes, dados não publicados). Suiriri affinis é encontrado em praticamente todo o Cerrado, ocorrendo também no nordeste brasileiro e nas savanas do Amapá (ZiMMER et $a l ., 2001)$. A biologia de ambas as espécies é parcamente documentada, tendo sido esclarecidos apenas recentemente vários aspectos da sua biologia reprodutiva e sucesso reprodutivo (L. E. Lopes \& M. Â. Marini, dados não publicados). Até o momento inexistem estudos detalhados sobre a dieta e comportamento de forrageamento dos representantes do gênero.

Devido às suas semelhanças morfológicas e ao fato de ocorrerem em sintopia (ZimmER et al., 2001; L. E. Lopes, dados não publicados), estas espécies fornecem uma interessante oportunidade para se investigar os diferentes métodos de partição de recursos. Isso porque, de acordo com o princípio da exclusão competitiva, espécies com requerimentos ecológicos muito similares poderiam competir entre si em um grau tal que poderia resultar na exclusão do competidor mais fraco (HARDIN, 1960; DiAmond, 1978; Schoener, 1982). Segundo Cody (1985), dentre os métodos de partição de recursos utilizados por espécies sintópicas, merecem destaque a seleção do sítio de forrageamento dentro de um mesmo hábitat e a utilização diferencial de sítios de forrageamento, o que pode se dar por meio de diferentes métodos de forrageamento e/ou de adaptações morfológicas.

Os objetivos deste trabalho foram: descrever comparativamente o comportamento de forrageamento de S. affinis e $S$. islerorum e descrever a dieta de ambas as espécies com base na análise de conteúdos estomacais e nas observações de campo.

\section{MATERIAL E MÉTODOS}

Este estudo foi conduzido na Estação Ecológica de Águas Emendadas (ESECAE), localizada em Planaltina,

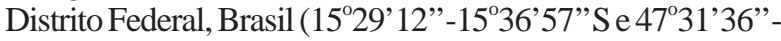
$\left.47^{\circ} 41^{\prime} 19^{\prime \prime} \mathrm{W}\right)$, que apresenta uma área de cerca de 10.500 ha. O clima é altamente sazonal e previsível, compreendendo de 1.500 a $1.750 \mathrm{~mm}$ de chuvas anuais, praticamente restritas ao período entre outubro e abril (NIMER, 1979). A vegetação da área amostrada é composta por um mosaico que abrange do campo limpo ao cerrado denso.

Foram realizadas observações no período compreendido entre janeiro de 2003 e janeiro de 2004. Para cada observação de forrageamento registrou-se, de acordo com Remsen JR. \& Robinson (1990): 1) comportamento de ataque; 2) direção do ataque, classificada em horizontal, vertical acima, vertical abaixo, diagonal acima e diagonal abaixo; 3) substrato atacado, classificado em folha verde, galho, flor, fruto, ar, solo; 4) 
distância percorrida durante o ataque (m); 5) altura em relação ao solo $(\mathrm{m}) ; 6$ ) espécie de planta da qual o ataque partiu; 7) densidade de folhagem no local de onde o ataque partiu, variando entre 0 (nenhuma folhagem) e 5 (folhagem extremamente densa); 8) fitofisionomia onde o ataque ocorreu, classificada em cerrado ralo, cerrado típico, cerrado denso e campos (sensu RIBEIRo \& WALTER, 1998), esta última agrupando as formações abertas observadas na área. Devido ao pequeno tamanho de muitas presas, que normalmente não são sequer visualizadas, não foram feitas distinções entre ataques bem ou mal sucedidos. A grafia em língua portuguesa dos diferentes comportamentos de forrageamento segue proposta de Volpato \& MendonçA-Lima (2002). Para se verificar a associação a bandos mistos de ambas as espécies ao longo do ano, cada vez que um casal/grupo de Suiriri foi acompanhado registrou-se se ele forrageava só ou acompanhado de outras espécies. As observações para cada espécie ocorreram de maneira independente.

Embora as observações seqüenciais proporcionem uma amostragem consideravelmente maior, as amostras geradas não são independentes entre si, levando a uma estimativa inacurada da proporção de cada comportamento empregado, bem como da variância associada (SAllie et al., 1990). Dessa maneira, com o intuito de se reduzir a pseudo-replicação devido à autocorrelação das amostras, só foram registradas observações de forrageamento separadas por um intervalo mínimo de $5 \mathrm{~min}$. Devido à grande mobilidade de ambas as espécies, este período foi assumido como suficiente para proporcionar observações independentes. $\mathrm{O}$ pequeno número de observações registradas diariamente para cada espécie, raramente mais que cinco, também contribuiu para a redução da pseudo-replicação.

Para a verificação da dieta dos representantes do gênero foram registrados os dados sobre conteúdo estomacal anotados nas etiquetas de espécimes depositados no Field Museum of Natural History (FMNH, 2003), em Chicago, e no Museu Nacional (MNRJ), no Rio de Janeiro. Informações sobre a dieta de $S$. suiriri também foram incluídas. Os seguintes espécimes trazem anotados dados relativos à sua dieta: S. affinis (FMNH 63431 a 63434 e 335154; MNRJ 13877, 13878, 13880 a 13883, H-963 e H-965); S. islerorum (FMNH 63435; MNRJ 13879); $S$. suiriri (FMNH 335151 a 335153; MNRJ 12880, 12881 e 32254). A inclusão de informações provenientes de etiquetas foi decidida após a investigação de REMSEN JR. et al.(1993) sobre a acurácia destas, que se mostraram notavelmente precisas. Os conteúdos estomacais de cinco espécimes de $S$. affinis depositados na Coleção Ornitológica Marcelo Bagno, da Universidade de Brasília, também foram analisados (COMB 0760, 0761, 0788, 1307 e sem número).

Para testar a existência de possíveis diferenças entre as variáveis medidas utilizou-se o teste U de MannWhitney para as variáveis contínuas, pois a distribuição dos dados não foi normal. Para as variáveis categóricas utilizou-se o teste do $\chi^{2}$, sendo agrupadas as categorias com freqüência inferior a 5\%. Todos os testes foram realizados de acordo com ZAR (1996) e conduzidos no Software Statistica (STATSOFT, 1995), adotando-se um nível de significância igual a $5 \%$.

\section{RESULTADOS}

Foram registradas 188 observações de forrageamento para $S$. affinis e 150 para $S$. islerorum. Diferenças significativas foram encontradas entre as duas espécies em relação à fitofisionomia e à densidade da folhagem no local do ataque, altura e substrato de forrageamento (Figs. 1-4). Contudo, não foram encontradas diferenças entre o comportamento de forrageamento, direção de ataque, distância de vôo e espécie de planta (Figs. 5-8).

A ingestão de água foi observada uma única vez. Em 12 de dezembro de 2003, às 13:40 h, um S. affinis adulto desceu ao solo e esgravatou as folhas caídas de uma Vochysia rufa Mart. (Vochysiaceae), bebendo a água acumulada na camada inferior do folhiço. Este foi um dos dias mais quentes do ano, sendo registrada uma temperatura máxima de $35^{\circ} \mathrm{C}$ (INMET, 2004). Esta também foi a única ocasião em que um $S$. affinis foi observado pousado no chão. Já $S$. islerorum frequientemente captura pequenos artrópodes no solo nu, porém nunca penetrando em meio ao capim. Em uma ocasião, um filhote com cerca de 90 dias de vida foi observado deslocandose por meio de pequenos saltos por mais de $50 \mathrm{~m}$ ao longo de uma estrada de terra.

Suiriri affinis foi observado associado em bandos mistos entre dezembro e maio ( $n=160$ casais/grupos observados, Fig. 9), sendo a composição dos bandos (n = 29) assim resumida: exclusivamente Lepidocolaptes angustirostris (Vieillot, 1818) (66\%), exclusivamente Picoides mixtus (Boddaert, 1783) (10\%) e ambas as espécies (21\%). Em um único caso também foi observada a associação de um grupo de Neothraupis fasciata (Lichtenstein, 1823) ao bando já contendo L. angustirostris e P. mixtus (3\%). Suiriri islerorum $(\mathrm{n}=167)$ apenas raramente se associou a bandos mistos, sendo todos os registros para o mês de janeiro (Fig. 10), sempre tendo como espécie núcleo $S$. affinis $(n=6)$. A associação de $S$. affinis a bandos mistos se deu com uma frequiência significativamente maior do que S. islerorum $\left(\chi^{2}\right.$ $=18,01 ; \mathrm{g} .1 .=1 ; \mathrm{p}<0,001)$.

Todos os 26 conteúdos estomacais de representantes do gênero examinados apresentaram exclusivamente artrópodes. Destes, 18 correspondiam a $S$. affinis, dois a $S$. islerorum e seis a $S$. suiriri. O conteúdo de cinco estômagos de $S$. affinis são analisados com maior detalhe na Tabela I.

O consumo de frutos por S. affinis e S. islerorum é bastante reduzido. Suiriri affinis foi registrado consumindo os frutos de Curatella americana L. (Dilleniaceae) (observação de ZIMMER et al., 2001) e Davilla elliptica A.St.-Hil. (Dilleniaceae), Phthirusa ovata (DC.) Eichler (Loranthaceae), Miconia pohliana Cogn. (Melastomataceae), Morus nigra L. (Moraceae, apenas no campus da UnB), Ouratea hexasperma (A.St.Hil.) Baill. (Ochnaceae) e Palicourea rigida H.B. \& K. (Rubiaceae). Já $S$. islerorum foi registrado ingerindo os frutos de Byrsonima coccolobifolia H.B. \& K. (Malpighiaceae), P. ovata e $O$. hexasperma. O único fruto ingerido habitualmente foi o de $O$. hexasperma, sendo todos os demais consumidos em menos de cinco ocasiões. Em uma observação ocasional foi registrada a ingestão de néctar das flores do pequizeiro, Caryocar brasiliense Camb. (Caryocaraceae), por S. affinis. 

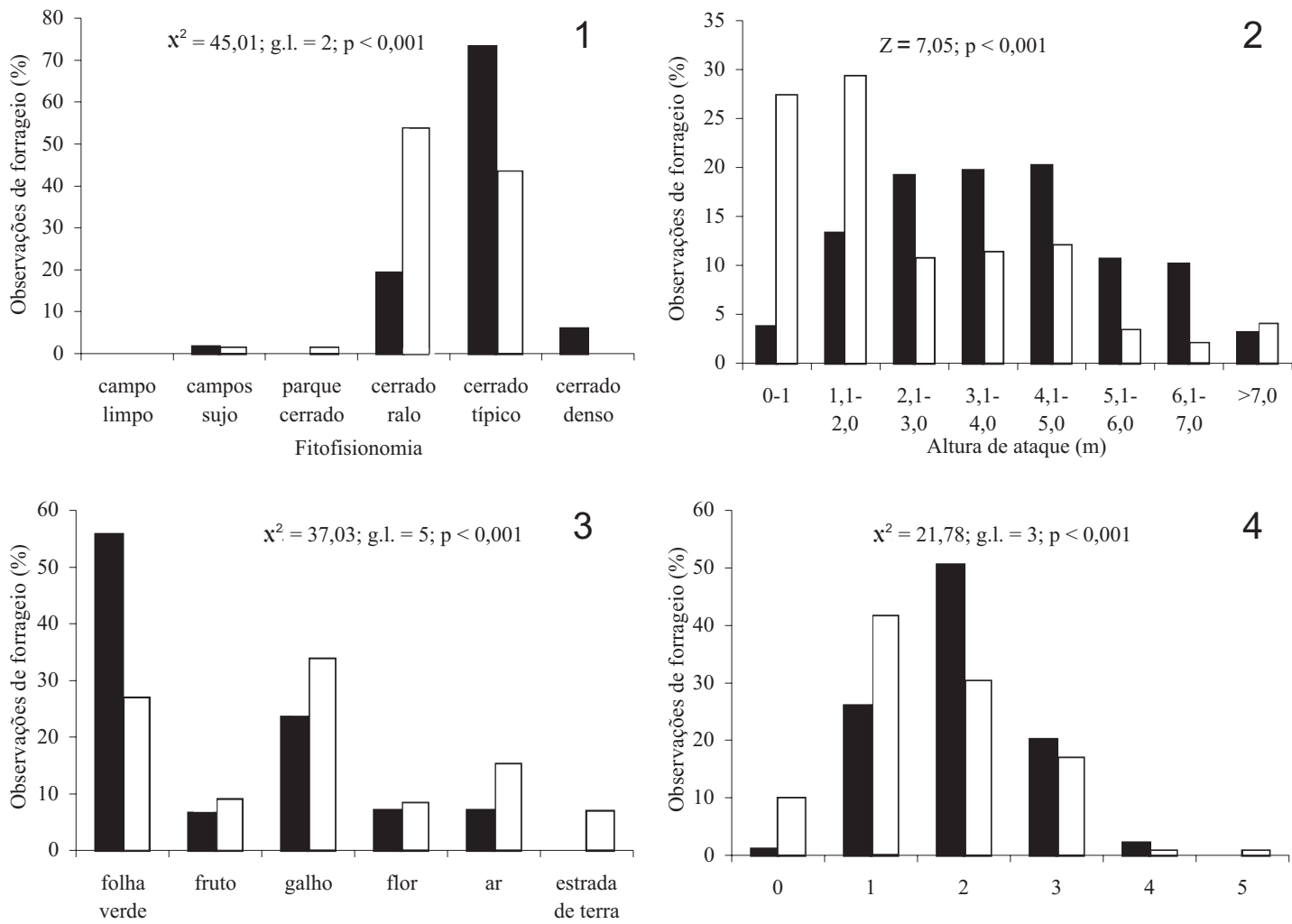

Substrato de ataque

Densidade de folhagem

Figs. 1-4. Porcentagem das observações de forrageamento de S. affinis (barras escuras) e S. islerorum (barras claras) para cada uma das variáveis que apresentaram diferenças significativas, na Estação Ecológica de Águas Emendadas, DF. (1, fitofisionomia; 2, altura de ataque; 3 , substrato de ataque; 4 , densidade de folhagem).
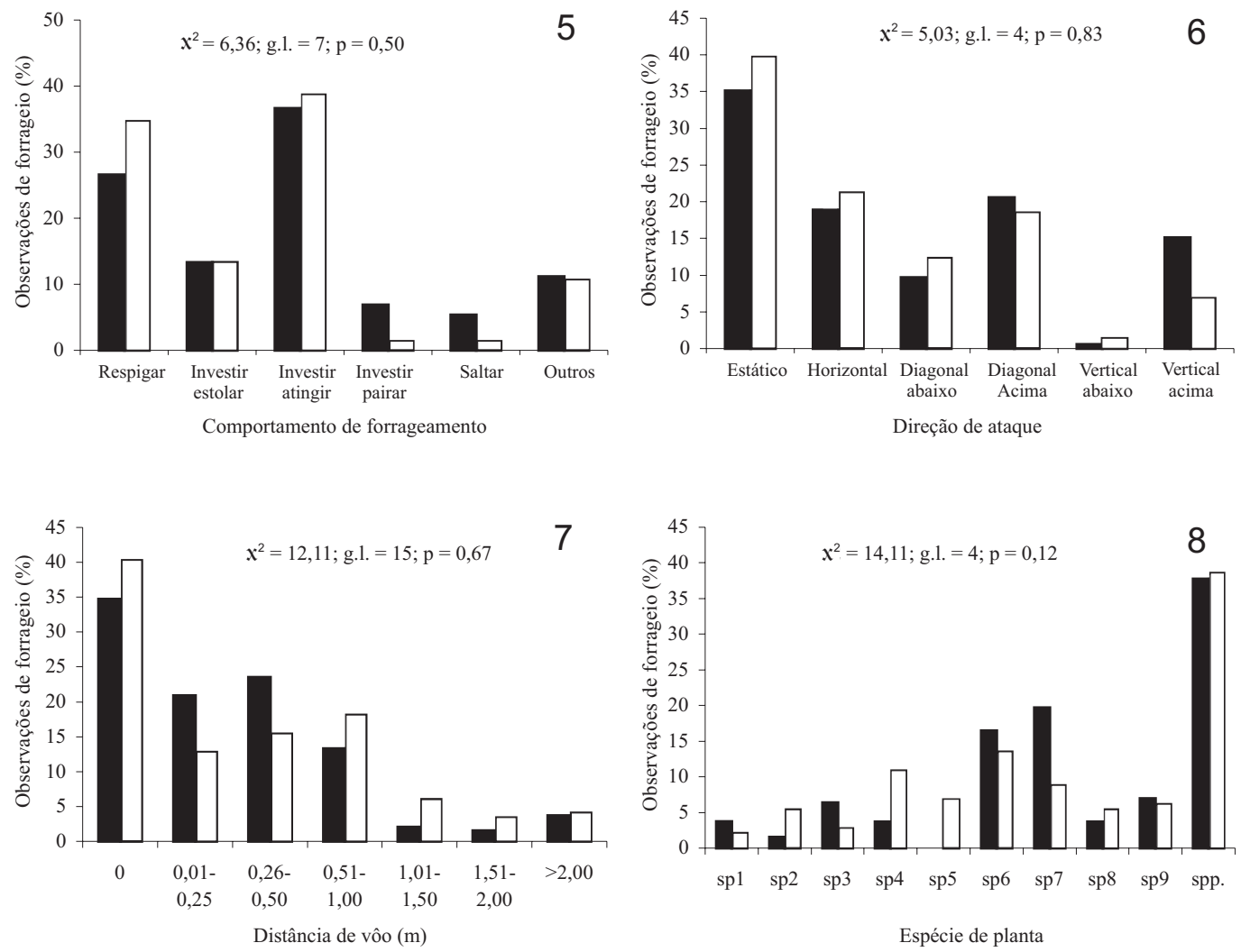

Figs. 5-8. Porcentagem das observações de forrageamento de S. affinis (barras escuras) e S. islerorum (barras claras) para cada uma das variáveis que não apresentaram diferenças significativas, na Estação Ecológica de Águas Emendadas, DF. (5, comportamento de forrageamento; 6 , direção de ataque; 7 , distância de ataque; 8 , espécie de planta). 


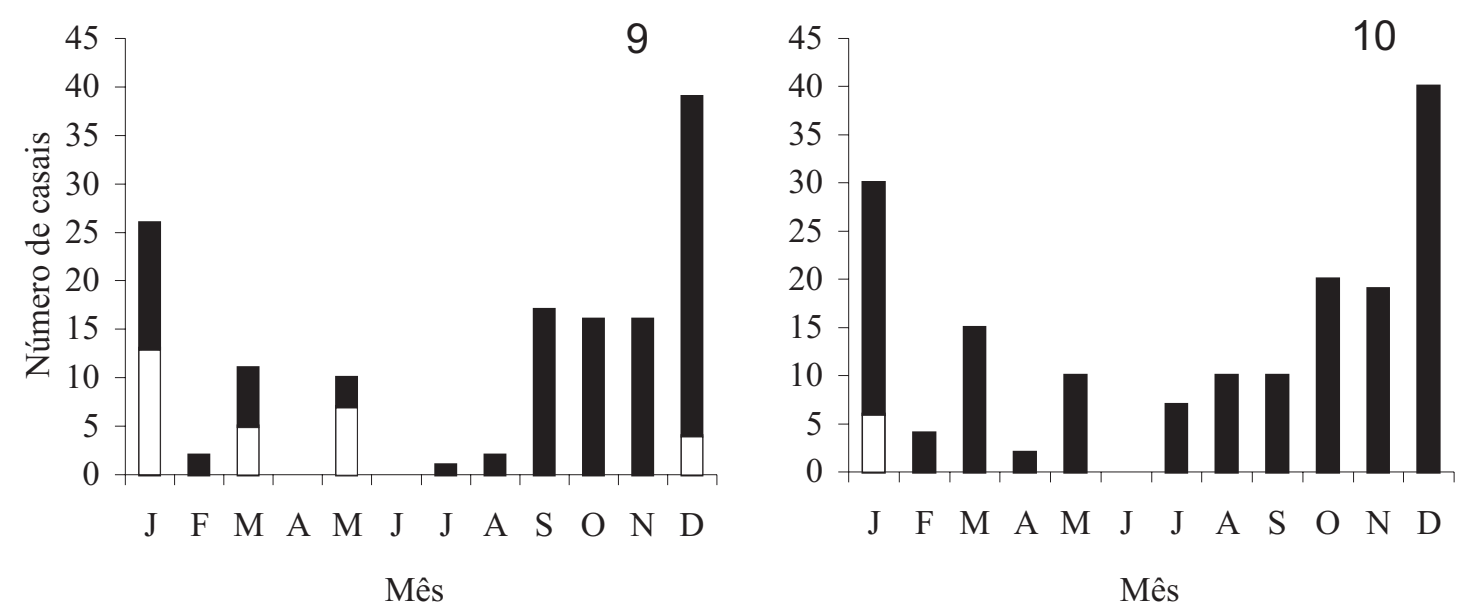

Figs. 9-10. Número de casais/grupos observados associados (barras claras) e não associados (barras escuras) a bandos mistos na Estação Ecológica de Águas Emendadas, DF. A amostragem não foi padronizada ao longo do ano. 9, Suiriri affinis. 10, Suiriri islerorum.

Tabela I. Artrópodes identificados em cinco conteúdos estomacais de Suiriri affinis (A, adulto; L, larva).

\begin{tabular}{llllllr}
\hline & \multicolumn{7}{c}{$N^{\circ}$ do conteúdo estomacal } & \\
\hline Artrópodes & 1 & 2 & 3 & 4 & 5 & Total \\
\hline Orthoptera & 1 & & & & 1 & 2 \\
Hemiptera & 4 & & 4 & 2 & & 10 \\
Homoptera & 2 & & & & 3 & 5 \\
Coleoptera & 4 & 2 & 1 & 1 & 4 & 12 \\
Diptera & 1 & & & & & 1 \\
Lepidoptera (A) & 1 & & & & & 1 \\
Lepidoptera (L) & 1 & & & & & 1 \\
Hymenoptera & 2 & & 8 & & & 10 \\
Formicidae & 1 & & & 1 & & 3 \\
Aranea & 2 & & & & 1 & \\
\hline
\end{tabular}

\section{DISCUSSÃO}

Ambas as espécies utilizaram basicamente duas táticas de forrageamento, que são o respigar (glean) e o investir-atingir (sally-strike). Suiriri affinis atacou $37 \%$ das suas presas respigando, enquanto $S$. islerorum utilizou-se desta técnica em $41 \%$ dos ataques. Estas observações diferem do encontrado para "S. affinis" por FitZPATRICK (1980), também no Distrito Federal, antes da descrição de S. islerorum. FITZPATRICK (1980) classificou "S. affinis" como um respigador especializado, que se utilizou desta técnica em $79 \%$ das observações. Uma possível explicação para parte destas diferenças é o fato de que este autor utilizou observações seqüenciais, inadequadas para estimar a proporção de cada comportamento empregado (SALLIE et al., 1990).

De acordo com os princípios da exclusão competitiva, para que duas espécies morfologicamente semelhantes coexistam são esperadas diferenças entre os seus nichos (MacArthur, 1958; Hardin, 1960; DiAmond, 1978). As análises aqui conduzidas demonstraram claras diferenças entre o comportamento de forrageamento de ambas, indicando que $S$. affinis forrageia em locais mais altos e de vegetação mais fechada que $S$. islerorum, sendo também observadas diferenças quanto ao tipo de substrato utilizado por cada uma das espécies. Diferenças entre a morfologia do bico são também observadas, sendo a razão entre o tamanho do bico de S. affinis e S. islerorum igual a 1,2 (ZimMER et al., 2001), dentro do limite de 1,2 a 1,4 estimada para o tamanho do bico de espécies de aves congenéricas e simpátricas (Hutchinson, 1959; Schoener, 1965). Assim, S. affinis e S. islerorum se utilizariam durante o forrageamento de diferentes: 1) partes de um mesmo hábitat; 2) táticas para a obtenção de alimento e 3) adaptações morfológicas (tamanho e formato do bico).

A exclusividade de artrópodes observada nos conteúdos estomacais analisados, associada ao baixo consumo de frutos registrado nas observações de forrageamento (7\% para S. affinis e 9\% para S. islerorum), revela uma dieta insetívora. O predomínio de Coleoptera, Hymenoptera e Hemiptera entre as presas capturadas é um fato bastante comum entre passeriformes insetívoros neotropicais (Lopes et al., 2005). Grupos de S. affinis foram observados em pelo menos uma dezena de ocasiões capturando insetos em arbustos de Miconia pohliana carregados de frutos maduros, sem, no entanto, consumilos. Outros exemplos de frutos ornitocóricos abundantes na área e pouco ou mesmo não consumidos são: Erythroxylum spp., Davilla elliptica A.St.-Hil., Didymopanax macrocarpum Seem. (Araliaceae), Psittacanthus robustus Mart. (Loranthaceae) e Miconia ferruginata DC. (Melostomataceae). Todos esses frutos foram avidamente disputados por outras espécies de Tyrannidae, tais como Tyrannus savana Vieillot, 1808, Xolmis cinerea (Vieillot, 1816), Elaenia chiriquensis Lawrence, 1865 e E. cristata Pelzeln, 1868. O consumo apenas eventual de frutos é largamente disseminado entre os Tyrannidae insetívoros, que assim complementam a sua dieta (FosTER, 1978).

Embora incomumente consumido pelas espécies estudadas, o néctar do pequizeiro parece ser um importante recurso alimentar no Cerrado, sendo avidamente consumido por diversas espécies, tais como Cypsnagra hirundinacea (Lesson, 1831), N. fasciata e Cyanocorax cristatellus (Temminck, 1823) (obs. pessoal). A importância desse recurso durante a estação seca no Cerrado é destacada por Melo (2001).

O papel de $S$. affinis como espécie nuclear nos bandos mistos no Distrito Federal, ao lado de N. fasciata, 
já havia sido destacada por Alves \& CAVAlCANTI (1996), que a registraram em $60 \%$ dos bandos mistos acompanhados. Já no Estado de São Paulo, esta espécie tem um papel apenas secundário, sendo registrada em apenas $13 \%$ dos bandos, que teriam como líderes $C$. hirundinacea e $N$. fasciata (RAgusa-NetTo, 2000). Embora estes dois trabalhos tenham sido publicados antes da descrição de S. islerorum, J. Ragusa-Netto (comunic. pess.) e M. A. S. Alves (comunic. pess.) relataram que, aparentemente, apenas $S$. affinis estaria envolvido nessas observações. O comportamento de $S$. affinis descrito por Alves \& CaVAlCANTI (1996) é também muito semelhante ao observado neste trabalho, sendo, portanto, a espécie em questão assumida como $S$. affinis. Embora $N$. fasciata e, em menor escala, C. hirundinacea sejam comuns na área de estudo (obs. pessoal), estas espécies normalmente não se associaram aos bandos liderados por S. affinis.

Embora a associação de $S$. islerorum aos bandos mistos só tenha sido registrada durante o mês de janeiro, é provável que este tipo de interação se estenda a outros meses, podendo ter passado despercebida em algumas ocasiões. Diferentemente do observado para $L$. angustirostris e $P$. mixtus, a associação de $S$. islerorum aos bandos mistos é bastante discreta e realizada a uma maior distância, o que a torna por vezes difícil de ser detectada.

Agradecimentos. À administração da ESECAE/ SEMARH por permitir o desenvolvimento deste estudo na área. À CAPES, pela bolsa de estudos concedida ao autor durante parte deste estudo, e à UnB, pelo suporte financeiro. Parte do estudo foi financiada através da Bolsa de Produtividade em Pesquisa concedida pelo CNPq a Miguel Â. Marini. As autorizações de anilhamento foram concedidas pelo CEMAVE à Rita Surrage e Miguel Â. Marini. Carolyn Proença, do Depto. de Botânica da UnB, identificou as espécies vegetais. Jorge Nacinovic e Marcos Raposo (MNRJ) proporcionaram todas as facilidades para o exame dos espécimes depositados nas coleções sob seus cuidados. Ao Field Museum por disponibilizar informações sobre espécimes depositados na sua coleção. A Miguel Ẩngelo Marini, Luiz Gonzaga, Mauro Pichorim, Guarino Colli, José Ragusa-Netto e um revisor anônimo pelas críticas e sugestões apresentadas em uma versão anterior deste trabalho.

\section{REFERÊNCIAS BIBLIOGRÁFICAS}

Alves, M. A. S. \& Cavalcanti, R. B. 1996. Sentinel behavior, seasonality, and the structure of bird flocks in a Brazilian savanna. Ornitología Neotropical 7:43-51.

Cody, M. L. 1985. An introduction to habitat selection in birds. In: CoDY, M. L. ed. Habitat selection in birds. San Diego, Academic Press. p.3-56.

Diamond, J. M. 1978. Niche shifts and the rediscovery of interspecific competition. American Scientist 66:322-331.

FitzPAtRICK, J. W. 1980. Foraging behavior of Neotropical flycatchers. The Condor 82(1):43-57.

FMNH. 2003. Birds collection databases. Disponível em: $<\mathrm{ht} \mathrm{tp://fm} \mathrm{1.fie} 1 \mathrm{dmuseum}$. org/collections/ search.cgi?dest=birds> Acesso em: 2.05.2003.
Foster, M. S. 1978. Total frugivory in tropical passerines: a reapraisal. Tropical Ecology 19(2):131-154.

Hardin, G. 1960. The competitive exclusion principle. Science 131:1291-1297.

Hutchinson, G. E. 1959. Homage to Santa Rosalia, or why are there so many kinds of animals? The American Naturalist 93:145-159.

INMET. 2004. Instituto Nacional de Meteorologia Parâmetros meteorológicos do Distrito Federal. Disponível em: <http://www.inmet.gov.br> Acesso em: 5.02 .2004$.

Lopes, L. E.; Fernandes, A. M. \& Marini, M. Â. 2005. Diet of some Atlantic forest birds. Ararajuba 13(1):95-103.

MacArthur, R. H. 1958. Population ecology of some warblers of northeastern coniferous forest. Ecology 39(4):599619 .

Melo, C. 2001. Diurnal bird visiting of Caryocar brasiliense Camb. in central Brazil. Revista Brasileira de Biologia 61(2):311-316.

Nimer, E. 1979. Climatologia no Brasil. Rio de Janeiro, Instituto Brasileiro de Geografia e Estatística (IBGE), Superintendência de Recursos Naturais e Meio Ambiente (SUPREN). 421p. (Série Recursos Naturais e Meio Ambiente, 4)

Ragusa-Netto, J. 2000. Raptors and "campo-cerrado" bird mixed flocks led by Cypsnagra hirundinacea (Emberizidae: Thraupinae). Revista Brasileira de Biologia 60(3):461467.

Remsen JR., J. V. \& Robinson, S. K. 1990. A classification scheme for foraging behavior in terrestrial habitats. In: Morrison, M. L.; Ralph, C. J.; Verner, J. \& Jehl JR., J. R. eds Avian foraging: theory, methodology and applications. Lawrence, Cooper Ornithological Society. p.144-160. (Studies in Avian Biology, 13)

Remsen Jr., J. V.; Hyde, M. A. \& Chapman, A. 1993. The diet of Neotropical trogons, motmots, barbets and toucans. The Condor 95(1):178-192.

Ribeiro, J. F. \& Walter, B. M. T. 1998. Fitofisionomias do bioma Cerrado. In: SAno, S. M. \& Almeida, S. P. eds. Cerrado: ambiente e flora. Planaltina, Empresa Brasileira de Pesquisa Agropecuária (EMBRAPA). p.89-166.

Sallie, J. H.; Verner, J. \& Bell, G. W. 1990. Sequential versus initial observations in studies of avian foraging. In: Morrison, M. L.; Ralph, C. J.; Verner, J. \& Jehl JR., J. R. eds. Avian foraging: theory, methodology and applications. Lawrence, Cooper Ornithological Society. p.166-173. (Studies in Avian Biology, 13)

SchoenER, T. W. 1965. The evolution of bill size differences among sympatric congeneric species of birds. Evolution 19:189-213.

1982. The controversy over interspecific competition. American Scientist 70:586-594.

Silva, J. M. C. \& BATES, J. M. 2002. Biogeographic patterns and conservation in the South American Cerrado: A tropical savanna Hotspot. BioScience 52:225-233.

Statsoft, Inc. 1995. Statistica for Windows (Computer Program Manual). Release 5.0. Tulsa, Statsoft Inc.

Volpato, G. H. \& MendonçA-Lima, A. 2002. Estratégias de forrageamento: proposta de termos para a língua Portuguesa. Ararajuba 10(1):101-105.

ZAR, J. H. 1996. Biostatistical analysis. London, PrenticeHall. 662p.

Zimmer, K. J.; Whittaker, A. \& Oren, D. C. 2001. A cryptic new species of Flycatcher (Tyrannidae: Suiriri) from the Cerrado region of central South America. The Auk 118(1):56-78. 